\title{
Analyse minéralogique quantitative d'un échan- tillon de sol : utilisation des données concernant la composition chimique de l'échantillon
}

\author{
Ary BRUAND \& René PROST (*) \\ I.N.R.A., Service d'Etude des Sols et de la Carte pédologique de France, Centre de Recherches d'Orléans, \\ Ardon, F 45160 Olivet \\ (*) I.N.R.A., Station de Science du sol, route de St-Cyr, F 78000 Versailles
}

RÉSUME

\begin{abstract}
La présence, dans un échantillon de sol, de minéraux dont la composition chimique est mal connue rend difficile l'utilisation des résultats des analyses chimiques pour une analyse minéralogique quantitative. L'étude, à titre d'exemple, d'un échantillon composé de kaolinite, d'illite, de goethite et de quartz permet de montrer tout d'abord que l'on ne peut pas se limiter à l'utilisation des compositions chimiques tirées de la littérature. En effet, le calcul est, dans ce cas, très imprécis.

Par conséquent, il est nécessaire de mettre en œuvre des méthodes permettant de connaître la composition chimique moyenne des minéraux de l'échantillon analysé. C'est l'objet de la deuxième étape de cette étude. La validité du calcul est alors liée à celle des méthodes ayant permis d'améliorer la caractérisation minéralogique et de déterminer la composition chimique moyenne de l'échantillon.
\end{abstract}

Mots clés additionnels : Kaolinite, illite, goethite, quartz, dissolution sélective, séparation granulométrique, diffraction $R X$

\begin{abstract}
Quantitative determination of mineral content : use of sample chemistry.
Use of the results of chemical analyses for quantitative mineralogical studies of soils is complicated by the presence of minerals of poorly known chemical composition. Study of a sample composed of kaolinite, illite, goethite and quartz showed that one could not depend on the chemical compositions of these minerals (illite and goethite) given in the literature, for the results of the calculations were then very inaccurate. It was therefore necessary to use methods providing data on the mean chemical composition of the minerals in the sample studied, and this was the second stage of the study. Validity of the calculations then depends on that of the methods for mineralogical characterization and for determining the mean chemical composition of the sample. Results showed the necessity to obtain more data on mineralogical characterization of soil samples, especially for 2: 1 clay minerals and iron oxi-hydroxides even if they were present in small proportions.
\end{abstract}

Additional key words : Kaolinite, illite, goethite, quartz, selective dissolution, size fractionation, X-ray diffraction.

\section{INTRODUCTION}

Le comportement physico-chimique d'un échantillon de sol est lié à la nature de ses constituants mais aussi à leurs proportions respectives dans l'échantillon étudié. C'est pourquoi, l'analyse minéralogique nécessite la caractérisation des différents constituants minéraux présents et la détermination de leurs proportions dans le mélange. Le premier volet, qui concerne l'identification des $\mathrm{n}$ constituants présents dans un échantillon, a fait l'objet d'un précédent travail (BRUAND \& PROST, 1986). Quant à la détermination de leurs proportions massiques $Q_{j}(j=1,2, \ldots n)$, les nombreux travaux effectués montrent que l'on utilise de façon très générale le fait que la ième propriété $\mathrm{M}_{\mathrm{i}}$ déterminée pour le mélange peut s'écrire sous la forme d'une combinaison linéaire (PEARSON, 1978; WIEGMANN et al., 1982; HodgSON \& DudENEY, 1984 ; PAWLOSK1, 1985). Il est alors nécessaire que les contributions des constituants à cette propriété soient additives. Le calcul implique l'établissement du système de $\mathrm{n}$ équations indépendantes suivant :

$$
\sum_{j=1}^{i=n} a_{i j} \cdot Q_{j}=M_{i} \quad(i=1,2, \ldots, i, \ldots, n)
$$


où chaque $\mathrm{a}_{\mathrm{ij}}$ est le coefficient donnant la contribution $\mathrm{du}$ jème constituant pour la ième propriété du mélange (WIEGMANN et al., 1982). Cette démarche est peu employée en raison de la présence fréquente, dans un échantillon de sol, d'espèces minéralogiques mal définies.

En conséquence, une partie des coefficients $\mathbf{a}_{\mathrm{ij}}$ n'est connue que de façon approximative. La validité du calcul dépend alors de la différence existant, pour chaque $a_{i j}$, entre la valeur qu'on lui affecte et sa valeur réelle (PEARSON, 1978).

Dans ce qui suit, on se propose de faire le calcul des $Q_{j}$ en utilisant les propriétés $M_{i}$ qui résultent de l'analyse chimique et les $\mathrm{a}_{\mathrm{ij}}$ déterminés de deux manières différentes:

1) en prenant les compositions chimiques données par la littérature ;

2) en déterminant la composition chimique des constituants présents dans le mélange.

Ces deux étapes permettront de discuter la nécessité qu'il y a à connaître avec précision la composition chimique des différents minéraux présents dans un échantillon.

\section{MATÉRIEL ET MÉTHODES}

\section{A. Echantillon}

L'échantillon de sol sélectionné est une fraction $<2 \mu \mathrm{m}(\mathrm{Ech} .<2 \mu \mathrm{m})$ issue de l'horizon B d'un profil de sol de "terres d'Aubues " (BruAnd, 1985). Ces sols, développés sur les plateaux jurassiques calcaires à la limite sud-est du Bassin Parisien, ont été étudiés par BAIZE (1971 et 1972). L'identification des constituants minéraux a montré que Ech. $<2 \mu \mathrm{m}$ est constitué de kaolinite, d'illite, de goethite et de quartz (BRUAND \& PROST, 1986).

\section{B. Méthodes}

\section{Le système d'équations linéaires}

Le système de $n$ équations indépendantes (1) peut s'écrire sous la forme du produit de matrices :

$$
\left|\begin{array}{ccccc}
a_{11} & \ldots & a_{1 j} & \ldots & a_{1 n} \\
\cdot & & \cdot & & \cdot \\
\cdot & & \cdot & & \cdot \\
a_{i 1} & \ldots & a_{i j} & \ldots & a_{\text {in }} \\
\cdot & & \cdot & & \cdot \\
a_{n 1} & \ldots & a_{n j} & \ldots & \cdot \\
a_{n n}
\end{array}\right| \quad\left|\begin{array}{c}
Q_{1} \\
\cdot \\
\cdot \\
Q_{j} \\
\cdot \\
\cdot \\
Q_{n}
\end{array}\right|=\left|\begin{array}{c}
M_{1} \\
\cdot \\
\cdot \\
M_{i} \\
\cdot \\
M_{n}
\end{array}\right|
$$

ou, de façon plus condensée :

$$
\text { A. } \mathrm{Q}=\mathrm{M}
$$

avec A matrice carrée d'ordre n, Q et $\mathrm{M}$ deux matrices unicolonne.

La quantité du ième élément chimique déterminée pour le mélange donne la valeur du terme $M_{i}$ et la quantité de cet élément dans le jème constituant, celle du terme $a_{i j}$. La résolution du système consiste ensuite à utiliser les propriétés du calcul matriciel pour déterminer les termes de la matrice $\mathrm{Q}$.

\section{Déterminations des grandeurs nécessaires à la réso- lution du système d'équations}

Lors d'une première étape, les valeurs des termes $\mathbf{M}_{\mathrm{i}}$ ont été déterminées par l'analyse chimique de Ech. $<2 \mu \mathrm{m}$ et celles des coefficients $\mathrm{a}_{\mathrm{ij}}$, en utilisant la composition chimique des minéraux de la même famille donnés en référence dans la littérature. La teneur en $\mathrm{H}_{2} \mathrm{O}$ de constitution de Ech. $<2 \mu \mathrm{m}$ est déterminée à partir de la courbe de perte de masse en fonction de la température (analyse thermopondérale). A cet effet, une thermobalance ADAMEL est utilisée, la prise d'essai est de $500 \mathrm{mg}$ et la vitesse de chauffage de $150^{\circ} \mathrm{C}$ par heure. La courbe obtenue se décompose en 3 domaines de température limités chacun par 2 points d'inflexion subhorizontaux délimités à l'aide de la courbe dérivée (fig. 1). La matière organique ayant été préalablement détruite avec $\mathrm{H}_{2} \mathrm{O}_{2}$, le premier domaine (I) est attribué à l'eau d'hydratation des cations interfoliaires, le second (II) à la déshydroxylation des hydroxydes métalliques et pour le troisième (III), il s'agit de la déshydroxylation des minéraux argileux. L'analyse chimique totale de Ech. $<2 \mu \mathrm{m}$ est effectuée au laboratoire d'Analyse des Sols d'Arras. Si est dosé par fluorescence $X$ après préparation d'une solution solide par fusion au tétraborate de sodium, $\mathrm{Al}$ par émission (plasma induit), $\mathrm{Fe}, \mathrm{Mg}, \mathrm{Ca}, \mathrm{Na}$ et $\mathrm{K}$ par absorption atomique après mise en solution avec l'acide fluorhydrique en présence de $\mathrm{HClO}_{4}$. Les teneurs en éléments chimiques sont exprimées en \% massique d'oxyde rapporté à la masse de l'échantillon calciné $\left(1100^{\circ} \mathrm{C}\right)$. Par conséquent, les valeurs de $\mathrm{Q}_{\mathrm{j}}$ obtenues sont exprimées par rapport à ce même état de référence.

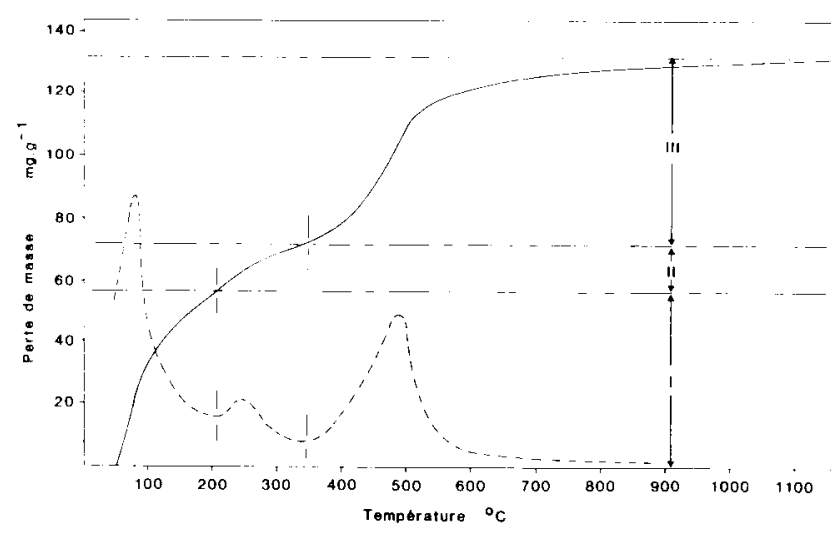

Figure 1

Courbe de la perte de masse de Ech. $<2 \mu \mathrm{m}$ en fonction de la température (- : courbe cumulée; -----. : courbe dérivée).

Mass loss curve of Lih. $<2 \mu m$ in relatom wilh temperature ( - : cumulative curve; ------ : derivative curve).

Cette première étape montre que l'illite est le constituant le plus représenté mais aussi celui pour lequel la constitution chimique est la plus mal connue.

Les traitements utilisés dans la deuxième étape doivent permettre de caractériser l'illite de Ech. $<2 \mu \mathrm{m}$ 
après avoir éliminé successivement les autres constituants. La séparation des particules en fonction de leur taille, après avoir saturé Ech. $<2 \mu \mathrm{m}$ avec $\mathrm{Na}^{+}$, permet d'éliminer le quartz de la fraction $<0,2 \mu \mathrm{m}$. La kaolinite est ensuite éliminée à l'aide d'une méthode qui consiste à faire bouillir l'échantillon dispersé dans de la soude 5 M (NORRISH \& TAYLOR, 1961 ; KAMPF \& SCHWERTMANN, 1982). Enfin, la goethite est dissoute à l'aide d'un mélange d'acide oxalique et d'oxalate d'ammonium à $\mathrm{pH}=3$, le mélange étant soumis à un rayonnement ultraviolet (DE ENDREDY, 1963 ; SCHWERTMANN, 1964 ; SCHWARZMANN \& SPARR, 1969). Ainsi, n'étant plus constitué que d'illite (BRUAND \& PROST, 1986), l'échantillon est mis sous forme calcique à l'aide d'une solution normale de $\mathrm{CaCl}_{2}$ avant que sa composition chimique ne soit déterminée à l'aide des méthodes analytiques précédemment décrites.

L'illite n'est pas le seul constituant de Ech. $<2 \mu \mathrm{m}$ pour lequel la constitution chimique donnée par la littérature varie beaucoup suivant les références, c'est aussi le cas de la goethite. En effet, on observe très fréquemment la présence d'aluminium dans le réseau cristallin, ce type de substitution peut concerner jusqu'à $32 \%$ des sites (FITZPATRICK \& SCHWERTMANN, 1982). La proportion du nombre d'atomes d'aluminium $\mathrm{x}$, $\mathrm{x}=\mathrm{N}_{\mathrm{Al}} /\left(\mathrm{N}_{\mathrm{Al}}+\mathrm{N}_{\mathrm{Fe}}\right)$, est déterminée à l'aide du paramètre $\mathrm{c}$ de la maille orthorhombique de la goethite à partir de la relation :

où

$$
\begin{gathered}
x=(1730-572,0 c) / 100 \\
c=\left[\left(1 / d_{111}\right)^{2}-\left(1 / d_{110}\right)^{2}\right]^{-1 / 2}
\end{gathered}
$$

(SCHUlze, 1984 ; CAMBIER, 1986). La valeur de x est déterminée après concentration des oxyhydroxydes de fer de Ech. $<2 \mu \mathrm{m}$ à l'aide de la méthode utilisant de la soude $5 \mathrm{M}$. Les valeurs de $\mathrm{d}_{110}$ et $\mathrm{d}_{111}$ sont obtenues en diffraction des $\mathrm{RX}$ en effectuant un comptage pas à pas durant 20 secondes chaque $1 / 100$ de ${ }^{\circ} 2 \theta$. La composition chimique des autres constituants présents dans l'échantillon étant connue, celle de la kaolinite apparaît alors comme déterminante pour la précision des calculs. En conséquence, sa composition chimique est déterminée à l'aide d'un microscope électronique JEOL JEM-100CX (modèle TEMSCAN) équipé d'un spectromètre $\mathrm{X}$ à sélection d'énergie (Université Catholique de Louvain ; Belgique).

Les particules analysées sont sélectionnées en fonction de leur morphologie après avoir dispersé Ech. $<2 \mu \mathrm{m}$ en milieu aqueux et l'avoir déposé sur une grille de nylon (RODRIQUE, 1980 et 1984).

\section{RÉSULTATS ET DISCUSSION}

Ech. $<2 \mu \mathrm{m}$ étant constitué de kaolinite, d'illite, de quartz et de goethite, le système d'équations s'écrit :

$$
\sum_{j=1}^{j=4} a_{i j} \cdot Q_{j}=M_{i} \quad(i=1,2,3,4)
$$

Le calcul est abordé en considérant les compositions chimiques d'échantillons référencés trouvées dans la littérature puis celles déterminées à partir des constituants séparés après avoir effectué des prétraitements adaptés.

\section{A. Résolution du système d'équations à l'aide de don- nées issues de la littérature}

Les valeurs des coefficients de variation calculées pour chaque élément, et pour les différentes kaolinites et illites de référence choisies, montrent que l'imprécision dépend de la nature du minéral et de l'élément chimique considérés (tabl. 1 et 2 ). Afin de réduire au maximum cette imprécision, il est nécessaire d'établir les 4 équations en choisissant les éléments pour lesquels les coefficients de variation sont les plus faibles.

Ainsi, si les proportions massiques de kaolinite, d'illite, de goethite et de quartz correspondent respectivernent à $Q_{1}, Q_{2}, Q_{3}$ et $Q_{4}$, le produit de matrices s'écrit :

$$
\left|\begin{array}{llll}
0 & 0 & \mathrm{a}_{13} & 0 \\
\mathrm{a}_{21} & \mathrm{a}_{22} & 0 & 0 \\
\mathrm{a}_{31} & \mathrm{a}_{32} & \mathrm{a}_{33} & 0 \\
\mathrm{a}_{41} & \mathrm{a}_{42} & 0 & \mathrm{a}_{44}
\end{array}\right| \quad\left|\begin{array}{l}
\mathrm{Q}_{1} \\
\mathrm{Q}_{2} \\
\mathrm{Q}_{3} \\
\mathrm{Q}_{4}
\end{array}\right|=\left|\begin{array}{l}
\mathrm{M}_{1} \\
\mathrm{M}_{2} \\
\mathrm{M}_{3} \\
\mathrm{M}_{4}
\end{array}\right|
$$

avec $\mathrm{M}_{1}$ et $\mathrm{M}_{2}$ teneurs en $\mathrm{H}_{2} \mathrm{O}$ de constitution de Ech. $<2 \mu \mathrm{m}$ attribuées respectivement à la goethite et aux minéraux argileux, $\mathrm{M}_{3}$ teneur en $\mathrm{Al}_{2} \mathrm{O}_{3}$ de Ech. $<2 \mu \mathrm{m}$ et $\mathrm{M}_{4}$ teneur en $\mathrm{SiO}_{2}$ de Ech. $<2 \mu \mathrm{m}$. Les valeurs de $\mathrm{M}_{1}$ et $\mathbf{M}_{2}$, déterminées à partir de la courbe de perte de masse en fonction de la température, sont respectivement de 1,7 et 7,0 (fig. 1, tabl. 3). La valeur de $\mathrm{M}_{3}$ est égale à 29,1 et celle de $\mathrm{M}_{4}$ à 53,4 (tabl. 4).

Quant à la détermination de la valeur des coefficients $\mathrm{a}_{\mathrm{ij}}$, elle implique que l'on choisisse une kaolinite et une illite de référence et que l'on attribue à la goethite présente dans Ech. $<2 \mu \mathrm{m}$ un taux de substitution de $\mathrm{Fe}$ par $\mathrm{Al}$. Or, comme indiqué plus haut, les données dont on dispose ne permettent pas de faire un tel choix. Par conséquent, le calcul qui suit est effectué en envisageant toutes les combinaisons possibles entre les illites et kaolinites de référence présentées dans les tableaux 1 et 2 . Néanmoins, pour la goethite, on a choisi de ne calculer que les solutions correspondant à 2 cas extrêmes de taux de substitution trouvés dans la littérature $(0$ et $32 \%)$ (FITZPATRICK \& SCHWERT. MANN, 1982) et à un cas intermédiaire (15\%) (tabl. 5).

\section{Calcul}

La somme des proportions massiques devant être proche de 1 pour que la solution soit acceptable, nous admettrons qu'une solution peut être retenue lorsque $\Sigma Q_{j}$ est compris entre 0,95 et 1,05 . Par ailleurs, chaque valeur de $\mathrm{Q}_{\mathrm{j}}$ doit être $>0$.

Les figures $2 \mathrm{a}, 2 \mathrm{~b}$ et $2 \mathrm{c}$ correspondent respectivement à des taux de substitution de 0,15 et $32 \%$ pour la goethite (tabl. 5). Des symboles distincts permettent de différencier les solutions suivant l'illite de référence utilisée pour le calcul (tabl. 2). Par conséquent, pour une même figure et un même symbole, seule la kaolinite de référence utilisée varie (tabl. 1).

Le calcul des solutions du système d'équations (3) montre que lorsque le taux de substitution de Fe par Al dans la goethite est nul, les illites I.2, I.4 et I.5 condui- 
TABLEAU 1

Compositions chimiques de différentes kaolinites exprimées en \% de la masse de l'échantillon calciné (K.2, K.3 et K.4 : CAILLEERE et al., $1982 ; K .5:$ CAMBIER, 1976).

Chemical composition of different kaolinites expressed in $\%$ of the calcined mass sample (K.2, K.3 and K.4 : CAILLERE et al., $1982 ; K .5:$ CAMBIER, 1976).

\begin{tabular}{|c|c|c|c|c|c|c|c|c|c|}
\hline & $\mathrm{SiO}_{2}$ & $\mathrm{TiO}_{2}$ & $\mathrm{Al}_{2} \mathrm{O}_{3}$ & $\mathrm{Fe}_{2} \mathrm{O}_{3}$ & $\mathrm{MgO}$ & $\mathrm{CaO}$ & $\mathrm{Na}_{2} \mathrm{O}$ & $\mathrm{K}_{2} \mathrm{O}$ & $\mathrm{H}_{2} \mathrm{O}$ \\
\hline K.I Théorique & 54,1 & - & 45,9 & - & - & - & - & - & 16,3 \\
\hline K.2 La Chartreuse & 54,1 & - & 44,3 & 0,9 & Tr. & 0,7 & -- & - & 16,0 \\
\hline K.3 Lewistone & 52,7 & 0,5 & 46,0 & 0,1 & Tr. & Tr. & 0,2 & 0,5 & 15,7 \\
\hline K.4 Limoges & 54,1 & 2,8 & 43,1 & - & - & - & - & - & 15,1 \\
\hline K.5 St Austell & 53,9 & Tr. & 45,3 & 0,6 & 0,2 & - & - & - & 16,1 \\
\hline Teneur moyenne & 53,8 & - & 44,9 & - & - & - & - & - & 15,8 \\
\hline Ecart type & 0,6 & - & 1,1 & - & - & - & - & - & 0,4 \\
\hline Coefficient de variation & 1,0 & - & 2,4 & - & - & - & - & - & 2,6 \\
\hline
\end{tabular}

TABLEAU 2

Compositions chimiques d'illites exprimées en \% de la masse de l'échantillon calciné

(I.I et 1.2 : HOWER \& MOWATT, 1966; I.3: GAUDETTE, 1965; I.4, I.6 et I.7: ROBERT \& BARSHAD, 1972: I.5: ARVIEU \& CHAUSSIDON). Chemical composition of illite expressed in \% of the calcined sample weight

(I.1 and I.2: HOWER \& MOWATT, 1966; I.3: GAUDETTE, 1965; I.4, I.6 and I.7: ROBERT \& BARSHAD, I972; I.5: ARVIEU \& CHAUSSIDON).

\begin{tabular}{|c|c|c|c|c|c|c|c|c|c|c|}
\hline & $\mathrm{SiO}_{2}$ & $\mathrm{TiO}_{2}$ & $\mathrm{Al}_{2} \mathrm{O}_{3}$ & $\mathrm{Fe}_{2} \mathrm{O}_{3}$ & $\mathrm{FeO}$ & $\mathrm{MgO}$ & $\mathrm{CaO}$ & $\mathrm{Na}_{2} \mathrm{O}$ & $\mathrm{K}_{2} \mathrm{O}$ & $\mathrm{H}_{2} \mathrm{O}$ \\
\hline I. I Interlake $<0,5 \mu \mathrm{m}$ & 54,9 & - & 30,4 & 0,6 & 1,0 & 3,3 & 0,1 & 0,1 & 9,6 & 4,8 \\
\hline I.2 Silver Hill $<0,5 \mu \mathrm{m}$ & 58,2 & - & 23,3 & 5,5 & 1,3 & 2,9 & 0,2 & 0,1 & 8,5 & 4,8 \\
\hline I. 3 Burnt Bluff $<2 \mu \mathrm{m}$ & 57,3 & - & 27,1 & 0,9 & 1,4 & 3,9 & 0,5 & 0,2 & 8,7 & 4,8 \\
\hline I.4 Puy $<2 \mu \mathrm{m}$ & 54,3 & 0,4 & 23,2 & 6,7 & 0,5 & 4,9 & 1,1 & 0,2 & 8,7 & 4,7 \\
\hline I.5 Puy $<2 \mu \mathrm{m}$ & 53,8 & 0,4 & 22,0 & 7,5 & 0,4 & 4,9 & 2,5 & 0,5 & 8,0 & 4,7 \\
\hline I. 6 Fithiam $0,3-0,5 \mu \mathrm{m}$ & 55,6 & 0,6 & 26,6 & 4,5 & 2,3 & 2,3 & 1,0 & 0,6 & 6,5 & 4,8 \\
\hline I. 7 Grundite $0,3-0,5 \mu \mathrm{m}$ & 54,1 & 0,6 & 28,9 & 6,4 & 1,6 & 1,5 & 0,3 & 0,7 & 5,9 & 4,8 \\
\hline Teneur moyenne & 55,4 & - & 25,9 & 4,6 & 1,2 & 3,4 & 0,8 & 0,3 & 8,0 & 4,8 \\
\hline Ecart type & 1,6 & - & 2,9 & 2,6 & 0,6 & 1,2 & 0,8 & 0,2 & 1,2 & 0,1 \\
\hline Coefficient de variation & 2,8 & - & 11,3 & 56,5 & 50,7 & 35,5 & 78,2 & 66,6 & 15,3 & 1,0 \\
\hline
\end{tabular}

TABLEAU 3

Perte de masse de Ech. $<2 \mu m$ en fonction de la température (en \% de la masse de l'échantillon calciné).

Mass loss of Ech. $<2 \mu \mathrm{m}$ in relation with the temperature (in \% of the calcined sample mass).

\begin{tabular}{|c|c|c|}
\hline \multicolumn{3}{|c|}{ Domaines de température } \\
\hline$\stackrel{\text { I }}{\text { Jusqu'à } 210^{\circ} \mathrm{C}}$ & $\underset{210-350^{\circ} \mathrm{C}}{\mathrm{II}}$ & III \\
\hline 6,4 & 1,7 & 7,0 \\
\hline
\end{tabular}

TABLEAU 4

Composition chimique de Ech. $<2 \mu$ m exprimée en $\%$ de la masse de l'échantillon calciné.

Chemical composition of Ech. $<2 \mu \mathrm{m}$ expressed in \% of the calcined sample mass.

\begin{tabular}{cccccccc}
\hline \hline $\mathrm{SiO}_{2}$ & $\mathrm{Al}_{2} \mathrm{O}_{3}$ & $\mathrm{Fe}_{2} \mathrm{O}_{3}$ & $\mathrm{MgO}$ & $\mathrm{CaO}$ & $\mathrm{Na}_{2} \mathrm{O}$ & $\mathrm{K}_{2} \mathrm{O}$ & $\mathrm{H}_{2} \mathrm{O}$ \\
\hline 53,4 & 29,1 & 12,5 & 1,4 & 1,0 & 0,1 & 2,5 & 8,7 \\
\hline \hline
\end{tabular}

sent à des valeurs de $\mathrm{Q}_{4}<0$. De plus, aucune des solutions obtenues avec les autres illites ne satisfait la condition sur $\Sigma Q_{j}$ (fig. 2, tabl. 6). Lorsque le taux est de

\section{TABLEAU 5}

Composition chimique théorique d'une goethite non substituée, Al-substituée à $15 \%$ et $32 \%$.

Theoretical chemical composition of a non-substituted goethite, $15 \%$ and $32 \%$ Al-substituted.

\begin{tabular}{lccc}
\hline \hline & $\mathrm{Al}_{2} \mathrm{O}_{3}$ & $\mathrm{Fe}_{2} \mathrm{O}_{3}$ & $\mathrm{H}_{2} \mathrm{O}$ \\
\hline $\mathrm{Fe} \mathrm{OH} \mathrm{OH}$ & 0 & 100 & 11,2 \\
$\mathrm{Fe}_{0,85} \mathrm{Al}_{0,15} \mathrm{O} \mathrm{OH}$ & 10,1 & 89,9 & 11,9 \\
$\mathrm{Fe}_{0,68} \mathrm{Al}_{0,32} \mathrm{O} \mathrm{OH}$ & 23,1 & 76,9 & 12,7 \\
\hline \hline
\end{tabular}

$15 \%$, seules les solutions obtenues avec I.1, I.3 et I.7 le satisfont. Enfin, lorsqu'il est de $32 \%$, exceptées les solutions obtenues avec I.4 et I.5, toutes les autres la satisfont.

\section{Discussion}

Dans ces conditions, c'est-à-dire si l'on envisage toutes les solutions possibles et si l'on ne prend pas en compte la précision des déterminations lors de l'analyse chimique de Ech. $<2 \mu \mathrm{m}$, le calcul des proportions de chacun des constituants apparaît, d'ores et déjà, peu précis. Cette imprécision est due à la grande variabilité de composition chimique qui existe pour l'illite et la goethite présentes dans les échantillons de 

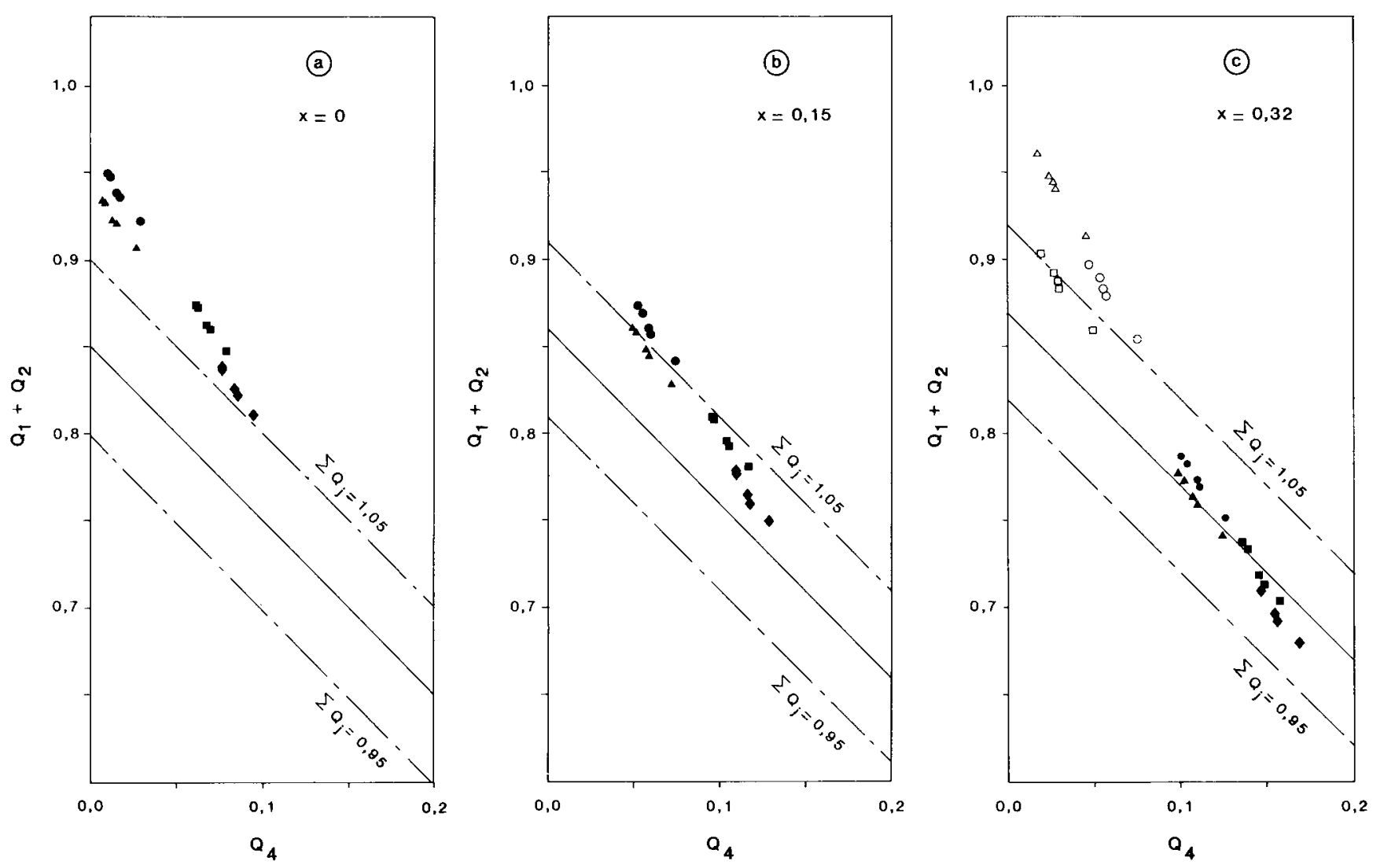

Figure 2

Représentation graphique des valeurs de $\left(Q_{1}+Q_{2}\right)$ et de $Q_{4}$ lorsque le taux de substitution de la goethite est de (a) $0 \%$, (b) $15 \%$ et (c) $32 \%$ (solutions obtenues avec:

$I .1: \diamond, \quad I .2: \square, \quad I .3: \wedge$

$I .4: \bigcirc, \quad I .5: \triangle, \quad I .6: \bullet, \quad I .7: \mathbf{\square}$

TABLEAU 6

Valeurs des proportions $Q_{j}$ en fonction du taux de substitution de la goethite $\left.10,95 \leqslant \Sigma Q_{j} \leqslant 1,05\right)$.

Values of $Q_{j}$ proportions in relation with the substitution rate of the goethite $\left(0,95 \leqslant \Sigma Q_{j} \leqslant 1,05\right)$.

\begin{tabular}{lcc}
\hline & \multicolumn{2}{c}{ Al-Substituée } \\
\cline { 2 - 3 } Non substituée & $\mathrm{x}=0,12$ & $\mathrm{x}=0,32$ \\
\hline & $0,26 \leqslant \mathrm{Q}_{1} \leqslant 0,32$ & $0,24 \leqslant \mathrm{Q}_{1} \leqslant 0,35$ \\
Pas de solution & $0,44 \leqslant \mathrm{Q}_{2} \leqslant 0,60$ & $0,34 \leqslant \mathrm{Q}_{2} \leqslant 0,66$ \\
$\mathrm{Q}_{\mathrm{j}}>1,05$ & $\mathrm{Q}_{3}=0,14$ & $\mathrm{Q}_{3}=0,13$ \\
& $0,05 \leqslant \mathrm{Q}_{4} \leqslant 0,13$ & $0,02 \leqslant \mathrm{Q}_{4} \leqslant 0,17$ \\
\hline
\end{tabular}

sol (fig. 2, tabl. 6). Il faut noter la grande sensibilité du calcul à la composition chimique de la goethite qui n'est pourtant présente qu'en faible proportion $\left(\mathrm{Q}_{3}\right.$ proche de 0,15$)$. En ce qui concerne la kaolinite, les variations de composition chimique recensées dans la littérature n'entraînent en comparaison que de faibles variations des valeurs des $Q_{j}$. L'écart entre les valeurs maximales et minimales est, en effet, toujours inférieur à 0,03 pour $Q_{1}, 0,04$ pour $Q_{2}$ et 0,02 pour $Q_{4}$ lorsque la composition chimique de la kaolinite est la seule à varier. Cette remarque rejoint celle de PEARSON (1978) sur l'importance de l'erreur faite lorsqu'un échantillon possède des minéraux argileux autres que la kaolinite.
Graphic representation of $\left(Q_{1}+Q_{2}\right)$ and $Q_{4}$ values with a (a) non substituted goethite, (b) $-15 \%$ and $(c)-32 \%$ Al-substituted goethite (results with:

I.I: •, I.2: $\square \quad$ I.3:

$1.4: \bigcirc, \quad 1.5: \triangle, \quad I .6: \bullet, \quad I .7: \mathbf{\square}$.

C'est pourquoi, dans l'étape qui suit et excepté pour le quartz, on s'attache à déterminer la composition chimique des différents minéraux de Ech. $<2 \mu \mathrm{m}$.

\section{B. Résolution du système d'équations après avoir déterminé la composition chimique de chaque cons- tituant de Ech. $<2 \mu \mathrm{m}$}

Après avoir soumis Ech. $<2 \mu \mathrm{m}$ à une succession de traitements permettant d'éliminer le quartz, la goethite et enfin la kaolinite, seule demeure l'illite (BRUAND \& PROST, 1986). La formule structurale moyenne déduite de l'analyse chimique (tabl. 7) est la suivante :

$$
\begin{aligned}
\left(\mathrm{Si}_{3,35}^{4} \mathrm{Al}_{0,65}^{3+}\right) \mathrm{O}_{10}^{2-}\left(\mathrm{Al}_{1,58}^{3}+\mathrm{Fe}_{0,20}^{3+} \mathrm{Mg}_{0,26}^{2+}\right)\left(\mathrm{OH}_{2}^{-}\right) \times \\
\times\left(\mathrm{K}_{0,50}^{+} \mathrm{Ca}_{0,13}^{2+}\right)
\end{aligned}
$$

Selon cette formule, 2,04 cavités octaédriques sur 3 sont occupées. La charge du feuillet est de $-0,79$ avec - 0,65 en couche tétraédrique et $-0,14$ en couche octaédrique. L'espace interfoliaire est occupé par des cations $\mathrm{K}^{+}$et $\mathrm{Ca}^{2+}$ qui représentent une charge totale $\mathrm{de}+0,76$.

Bien que la teneur en $\mathrm{K}_{2} \mathrm{O}$ soit un peu faible, de tels caractères sont ceux d'une illite (BRINDLEY \& BROWN, 1980).

Pour la goethite, les valeurs de $\mathrm{d}_{111}$ et $\mathrm{d}_{110}$ sont respectivement de $2,433 \AA$ et $4,153 \AA$ (fig. 3). Par consé- 
TABLEAU 7

Composition chimique des différents constituants de Ech. $<2 \mu \mathrm{m}$ (en \% de la somme des oxydes).

Chemical composition of the different components of Ech. $<2 \mu \mathrm{m}$ (in $\%$ of the total oxides).

\begin{tabular}{lcccccccc}
\hline \hline & $\mathrm{SiO}_{2}$ & $\mathrm{TiO}_{2}$ & $\mathrm{Al}_{2} \mathrm{O}_{3}$ & $\mathrm{Fe}_{2} \mathrm{O}_{3}$ & $\mathrm{MgO}$ & $\mathrm{CaO}$ & $\mathrm{K}_{2} \mathrm{O}$ & $\mathrm{H}_{2} \mathrm{O}$ \\
\hline Kaolinite & 52,1 & 0,6 & 44,7 & 1,9 & 0,1 & 0,3 & 0,3 & 16,3 \\
lllite & 54,1 & - & 30,5 & 4,3 & 2,8 & 2,0 & 6,3 & 4,8 \\
Goethite $(\mathrm{x}=0,13)$ & - & - & 8,7 & 91,3 & - & - & - & - \\
Quartz & 100 & - & - & - & - & - & - & - \\
\hline \hline
\end{tabular}
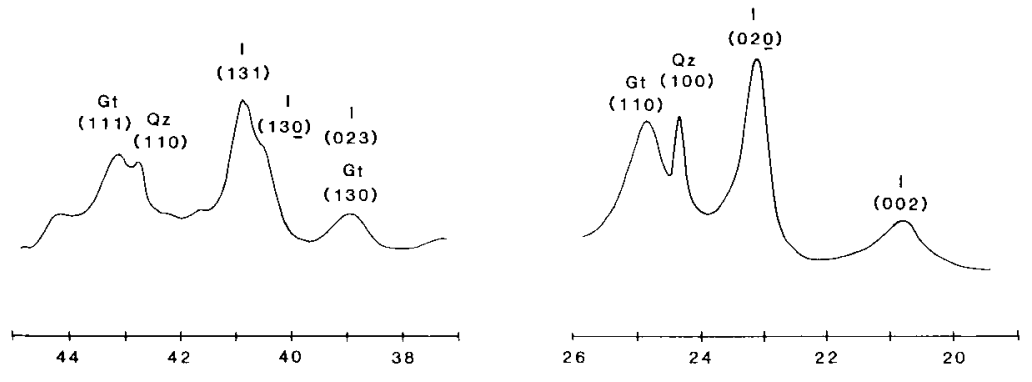

Figure 3

Diffractogramme $R X$ de Ech. $<2$ um sous forme de poudre après concentration de la goethite à l'aide du traitement utilisant $\mathrm{NaOH}$ $5 \mathrm{M}$. Position des raies ( $h \mathrm{kl}$ ) attribuées à la goethite (Gt), à l'illite (I) et au quartz $(Q z)$ dans les domaines $19-26^{\circ} 20$ et $37-45^{\circ} 20$.

$X$-ray powder diffraction diagram of Ech. $<2 \mu \mathrm{m}$ after concentration of goethite with $5 \mathrm{M} \mathrm{NaOH}$ treatment. Position of the (hkl) $X$ ray attributed to the goethite (Gt), the illite (I) and the quartz $(Q z)$ in the $19-26^{\circ} 20$ and $37-45^{\circ} 20$ range.

quent, le calcul conduit à $\mathrm{c}=3,002 \AA$ et la relation proposée par SCHULZE (1984) permet d'estimer la proportion du nombre d'atomes d'aluminium à $13 \%$ (tabl. 7).

Quant à la kaolinite, sa composition chimique obtenue en microanalyse à partir d'un dépôt sur grille de Ech. $<2 \mu \mathrm{m}$ est donnée dans le tableau 7.

\section{Calcul}

On peut calculer la solution du système d'équations (3) à l'aide des coefficients $a_{i j}$ correspondant à ces compositions chimiques. $\mathrm{M}_{1}$ et $\mathrm{M}_{2}$, teneurs en eau de constitution de Ech. $<2 \mu \mathrm{m}$ attribuées respectivement à la goethite et aux minéraux argileux, sont déterminées à partir de l'analyse thermopondérale et sont égales à $1,7 \pm 0,2 \%$ et $7,0 \pm 0,2 \% . \mathrm{M}_{3}$ et $\mathrm{M}_{4}$, respectivement teneur en $\mathrm{Al}_{2} \mathrm{O}_{3}$ et $\mathrm{SiO}_{2}$ de Ech. $<2 \mu \mathrm{m}$, sont par contre obtenues à partir de l'analyse chimique totale et égales à $29,1 \pm 0,1 \%$ et $53,4 \pm 0,1 \%$. $\mathrm{d}_{111}$ est estimé à $0,02^{\circ} 2 \theta$ près et $d_{110}$ à $0,03^{\circ} 2 \theta$. Par conséquent, le paramètre $\mathrm{c}$ de la maille orthorhombique de la goethite est connu à $0,002 \AA$ près. Ainsi, $x$ étant égal à $13 \pm 3 \%$, la valeur de $\mathrm{a}_{33}$ (teneur en $\mathrm{Al}_{2} \mathrm{O}_{3}$ de la goethite) est de $8,7 \pm 2,1 \%$ et celle de $\mathrm{a}_{13}$ (teneur en $\mathrm{H}_{2} \mathrm{O}$ de la goethite), de $11,8 \pm 0,1 \%$. Par ailleurs, on peut raisonnablement estimer que les valeurs des autres coefficients $a_{i j}$ sont connues à au moins $0,1 \%$ près. De la sorte, la solution est la suivante :

$$
\begin{aligned}
& \mathrm{Q}_{1}=0,28 \pm 0,05 \\
& \mathrm{Q}_{2}=0,50 \pm 0,09 \\
& \mathrm{Q}_{3}=0,14 \pm 0,02 \\
& \mathrm{Q}_{4}=0,12 \pm 0,04 .
\end{aligned}
$$

\section{Discussion}

La validité du calcul est liéé à celle des méthodes utilisées pour déterminer la composition chimique des différents minéraux de Ech. $<2 \mu \mathrm{m}$.

La succession de traitements ayant permis de connaître la formule chimique moyenne de l'illite, débute par un fractionnement granulométrique de Ech. $<2 \mu \mathrm{m}$. Celui-ci permet d'éliminer une fraction $2-0,2 \mu \mathrm{m}$ constituée de l'ensemble des particules de quartz mais aussi d'illite et de kaolinite. L'étude du gonflement interfoliaire en diffraction des RX ne révèle pas de différence de nature minéralogique entre les minéraux argileux de la fraction 2-0,2 $\mu \mathrm{m}$ et ceux de la fraction $<0,2 \mu \mathrm{m}$ (BRUAND \& PROST, 1986). La kaolinite de la fraction $<0,2 \mu \mathrm{m}$ est ensuite éliminée à l'aide du traitement qui utilise $\mathrm{NaOH} 5 \mathrm{M}$ (KAMPF \& SCHWERTMANN, 1982). Bien qu'une partie des particules d'illite soient aussi dissoutes, on a montré précédemment que l'illite, présente à l'issue de ce dernier traitement, est représentative de la phase illitique de Ech. $<2 \mu \mathrm{m}$ (BRUAND \& PROST, 1986). Quant à la dernière étape, elle vise à dissoudre la goethite de la fraction $<0,2 \mu \mathrm{m}$ en utilisant un mélange d'acide oxalique et d'oxalate d'ammonium en présence d'un rayonnement U.V. (DE ENDREDY, 1963 ; SCHWARZMANN \& SPARr, 1969). Ce traitement peut, en outre, extraire une partie du fer du réseau des minéraux argileux 2:1 (ROBERT \& TESSIER, 1974; JEANROY, 1983 ; CAMBIER, 1986). Par contre, le traitement qui permet de dissoudre la goethite et qui utilise un mélange complexant réducteur de dithionite et de citrate, permettrait d'extraire moins de fer du réseau des minéraux argileux mais, semble-t-il, lorsqu'il s'agit de $\mathrm{Fe}^{2+}$ (Jeanroy, 1983). Or, dans le cas de Ech. $<2 \mu \mathrm{m}$, l'étude de spectrométrie Mössbauer a montré 
que le fer, qu'il soit sous forme d'oxyhydroxyde ou dans le réseau des minéraux argileux, est à l'état de $\mathrm{Fe}^{3+}$ (BRUAND \& PROST, 1986). Le traitement de DE ENDREDY (1963) apparaît alors être le plus adapté. Quoi qu'il en soit, avec l'un ou l'autre réactif, seuls quelques $\%$ du fer présent dans le réseau des minéraux argileux serait extrait.

La composition chimique ainsi obtenue pour l'illite correspond au pôle le plus alumineux des illites présentées dans le tableau 1. Par ailleurs, il s'agit de la composition chimique moyenne d'une population de particules d'argile pour lesquelles cette composition peut être notablement variable.

En effet, DuPLAY (1984) a montré sur des illites de gisement, considérées comme ayant une composition chimique très homogène, qu'il existe de grandes variations de composition lorsque celle-ci est déterminée sur des monoparticules d'argile. Or, cette hétérogénéité s'accroît encore dans les conditions physico-chimiques du milieu superficiel où les minéraux hérités des matériaux géologiques s'altèrent.

Le taux de substitution de Fe par Al dans la goethite est déterminé après l'avoir concentré dans Ech. $<2 \mu \mathrm{m}$ à l'aide de la méthode utilisant $\mathrm{NaOH} 5 \mathrm{M}$. Les travaux de KAMPF \& SCHWERTMANN (1982) ont montré que, en présence de quartz, la concentration de Si en solution est suffisante pour empêcher toute évolution des oxyhydroxydes de fer. Le traitement chimique préalable à l'analyse est donc ici sans effet sur le minéral que l'on désire concentrer dans le mélange.

Quant à la composition chimique de la kaolinite, elle est déterminée en microscopie électronique à transmission à l'aide d'un spectromètre $\mathrm{X}$ à sélection d'énergie. Les particules analysées présentent une morphologie caractéristique qui permet de les identifier comme étant des particules de kaolinite. Cette façon de procéder conduit à analyser une population de particules qui n'est vraisemblablement pas tout à fait représentative de l'ensemble des particules de kaolinite. Comme pour l'illite, il en résulte une imprécision supplémentaire dont il n'a pas été tenu compte dans le calcul des proportions. La teneur en $\mathrm{Fe}_{2} \mathrm{O}_{3}$ est élevée mais légère- ment inférieure à celles obtenues pour des kaolinites ferrifères de sols tropicaux (JANOT et al., 1973 ; HERBILLON et al., 1976).

\section{CONCLUSION}

L'étude d'un échantillon de sol nous a permis de montrer que l'on ne peut pas se limiter à l'utilisation des constitutions chimiques tirées de la littérature pour des minéraux tels que l'illite et la goethite. Le cas de l'illite est celui rencontré pour de nombreux autres minéraux argileux $2: 1$ dont la composition chimique varie beaucoup suivant la référence choisie.

Quant à la goethite, bien qu'elle soit présente en faible proportion, sa composition chimique, liée au taux de substitution de Fe par Al, est apparue déterminante pour le calcul. Dans l'un et l'autre cas, la composition chimique a été déterminée à l'aide d'une combinaison de traitements visant, soit à séparer un ou plusieurs constituants du mélange, soit à les dissoudre sélectivement pour analyser ultérieurement le résidu de dissolution et/ou les éléments présents dans la solution obtenue. Indépendamment de la validité des traitements mis en œuvre, cette étude fait apparaître clairement que l'analyse minéralogique quantitative d'un échantillon de sol, à partir des résultats de l'analyse chimique, nécessite une connaissance précise de la composition chimique de chacun des constituants minéraux.

Recu le II juillet 1986. Accepté le 4 octobre 1987.

\section{REMERCIEMENTS}

Les auteurs remercient Messieurs G. SOIGNET et H. CielsiElsKK du Laboratoire d'Analyse des Sols (I.N.R.A.-Arras), Monsieur M. Hardy et Madame B. Le Berre du Service d'Etude des Sols et de la Carte Pédologique de France (I.N.R.A.-Ardon) ainsi que Monsieur R. Rodrique de l'Université Catholique de Louvain (Belgique), pour leur aide au cours de cette étude.

\section{RÉFÉRENCES BIBLIOGRAPHIQUES}

Arvieu J. C., Chaussidon J., 1964. Etude de la solubilisation acide d'une illite : extraction du potassium et évolution du résidu. $A n n$. agron., 15 (3), 207-229.

Baize D., 1971. Contribution à l'etude des sols des plateaux jurassiques de Bourgogne. Thèse de $3^{\mathrm{e}}$ cycle. Univ. Paris, $156 \mathrm{p}$.

Baize D., 1972. les sols développés dans la couverture des plateaux jurassiques de Bourgogne, Pédogenèse et origine. Sci. Sol., 1, 37-43.

Brindley G. W., Brown G., 1980. Crystal structures of clay minerals and their X-ray identification. Miner. Soc. Great Britain, Monograph. 5, London, 495 p.

Bruand A., 1985. Contribution à l'étude de la dynamique de l'organisation de matériaux gonflants. Application à un matériau provenant d'un sol argilo-limoneux de l'Auxerrois. Thèse de $3^{c}$ cycle, Univ. Paris VII, $225 \mathrm{p}$.

Bruand A., Prost R., 1986. Apport des méthodes d'enrichissement sélectives et des spectrométries à l'identification des constituants minéraux d'un échantillon de sol. Agronomie, 6 (8), 717-726.

Caillère D., Henin S., Rautureau M., 1982. Mineralogie des argiles. Classification et nomenclature. Tome 2, Masson Ed., 189 p.
Cambier Ph., 1976. Les associations kaolinite-oxyde de fer : cristallinité et propriétés de surface. D.E.A. Univ. Paris VI, 23 p.

Cambier Ph., 1986. Organisation des constituants et interactions physicochimiques au sein des microagrégats de sols. Application au système ferrallitique kaolinite-oxyde de fer. Thèse Doc. Univ. Paris VI, 226 p.

De Endredy A. S., 1963. Estimation of free iron oxides in soils and clays by a photolytic method. Clay Miner. Bull., 29 (5), 209-217.

Duplay J., 1984. Analyses chimiques ponctuelles d'argiles. Relations entre variations de composition dans une population de particules et température de formation. Sci. Geol. Bull., 37 (4), 307-317.

Fitzpatrick R. W., Schwertmann U., 1982. Al-substituted goethite. An indicator of pedogenic and other weathering environments in South Africa. Geoderma, 27, 335-347.

Gaudette H. E., 1965. Illite from Fond du Lac County, Wisconsin. Am. Mineral., 50, 411-417.

Herbillon A., Mestdagh M. M., Vielvoye L., Derouane E. G., 1976. Iron in kaolinite with special reference to kaolinite from tropical soils. Clay Miner., 11, 201-220. 
Hodgson M., Dudeney A. W. L., 1984. Estimation of clay proportions in mixtures by X-ray diffraction and computerized chemical mass balance. Clays Clay Miner., 32 (1), 19-28

Hower J., Mowatt T. C., 1966. The mineralogy of illites and mixedlayer illite/montmorillonite. Am. Mineral., 51, 825-854.

Janot Ch., Gibert H., Tobias Ch., 1973. Caractérisation de kaolinites ferrifères par spectrométrie Mössbauer. Bull. Soc. fr. Mineral. Cristallogr., 96, 281-291.

Jeanroy E., 1983. Diagnostic des formes du fer dans les pédogenèses tempérées. Evaluation par les réactifs chimiques d'extraction et apports de la spectrométrie Mössbauer. Thèse Univ. Nancy I, 177 p.

Kampf N., Schwertmann U., 1982. The 5-M-NaOH concentration treatment for iron oxides in soils. Clays clay Miner, 30 (6), 401-408.

Norrish K., Taylor R. M., 1961. The isomorphous replacement of iron by aluminium in soil goethites. J. Soil Sci., 12, 294-306.

Pawloski G. A., 1985. Quantitative determination of mineral content of geological samples by X-ray diffraction. Am. Miner., 70, 663-667.

Pearson M. J., 1978. Quantitative clay mineralogical analyses from the bulk chemistry of sedimentary rocks. Clays Clay Miner., 26 (6), 423-433.

Robert M., Barshad 1., 1972. Transformation expérimentale des micas en vermiculites ou smectites. Propriétés des smectites de transformation. Bull. Groupe fr. Argiles, 24, 137-151.

Robert M., Tessier D., 1974. Méthode de préparation des argiles de sols pour des études minéralogiques. Ann. agron., 25 (6), 859-882.

Rodrigue L., 1980. Utilisation d'un microscope électronique analytique par transmission pour l'identification de minéraux argileux. Mus. roy. Afr. centr., Tervuren (Belg.), Dept. Geol. Min., Rapp. ann., 1979, 57-67.

Rogrique L., 1984. Les minéraux d'amiante et leur caractérisation. Rev. Questions sci., 155 (2), 225-258.

Schulze D. G., 1984. The influence of aluminium on iron oxides. VIII. Unit-cell dimensions of Al-substituted goethites and estimation of $\mathrm{Al}$ from them. Clays clay Miner., 32 (11), 36-44.

Schwarzmann E., Sparr H., 1969. Die Wasserstoffbrückenbindung in Hydroxiden mit Diasporstruktur. Z. Naturforsch., 246, 8-11.

Schwertmann U., 1964. Differenzierung der Eisenoxide des Bodens durch photochemische Extraction mit saurer Ammonium-oxalatLösung. Z. Pflanzernernähr. Bodenk., 105, 194-202.

Wiegmann J., Horte C. H., Kranz G., 1982. Determination of the complete mineral composition of clays. Proc. VII Int. Clay Conf. 1981, Bologna and Pavia, Ed. H. Van Olphen and F. Veniale, Dev. Sed. 35, Elsevier, 365-372. 